\title{
A Validation Study of Liver Volumetry Estimation by a Semiautomated Software in Patients Undergoing Hepatic Resections
}

\author{
Suyash S. Kulkarni ${ }^{1,2}$ Nitin Sudhakar Shetty ${ }^{1,2}$ Kunal B. Gala ${ }^{1,2}$ Shraddha Patkar ${ }^{2,3}$ Amrita Narang \\ Ashwin M. Polnaya $^{4}$ Sushil Patil ${ }^{1}$ Neeraj G. Shetty ${ }^{1,2}$ Falguni Hota ${ }^{1,2}$ Mahesh Goel2,3 \\ 1'Interventional Radiology, Department of Radio-Diagnosis, Tata \\ Memorial Hospital, Tata Memorial Centre, Mumbai, Maharashtra, \\ India \\ ${ }^{2}$ Homi Bhabha National Institute, Mumbai, India \\ ${ }^{3}$ Gastrointestinal and HPB Surgery, Department of Surgical

\begin{abstract}
Address for correspondence Nitin Sudhakar Shetty, MD, EBIR, Interventional Radiology, Department of Radio-Diagnosis, Tata Memorial Hospital, Tata Memorial Centre, Dr. E. Borges Road, Parel, Mumbai, Maharashtra 400012, India
\end{abstract} \\ (e-mail: shettyns@tmc.gov.in; drnsshetty@gmail.com).
}

Oncology, Tata Memorial Hospital, Tata Memorial Centre, Mumbai, Maharashtra, India

${ }^{4}$ Department of Radio-Diagnosis and Imaging, A. J. Institute of

Medical Science and Research Centre, Mangalore, Karnataka, India

J Clin Interv Radiol ISVIR:2020;4:154-158

\begin{abstract}
Purpose The purpose of this study was to validate the use of a semiautomated software for liver volumetry preoperatively by comparing it with the volume of resected specimen in patients undergoing hepatic resections.

Materials and Methods This is a single-center retrospective study of patients who underwent estimation of future liver remnant (FLR) using Myrian XP-Liver which is a semiautomated software for hepatectomy. The estimated resection volume, which is the sum of volume of normal liver to be resected and tumor volume, was compared with actual specimen weight to calculate the accuracy of the software. The statistical analysis was performed with SPSS software version 24 .

Results Data on FLR estimation using the semiautomated software was available for 200 out of 388 patients who underwent formal hepatic resections. The median resected volume of surgical specimen was $650 \mathrm{~mL}$ (interquartile range [IQR] 364950), while the median estimated volume using the Myrian software was $617 \mathrm{~mL}$ (IQR 362-979). There was significant correlation between estimated resection volume calculated using the semiautomated method and actual specimen weight ( $p$-value $<0.0001$ ) with the Spearman's correlation value of 0.956 .

Conclusion The estimated volume of liver to be resected as calculated by the semi-

Keywords

- liver volumetry

- FLR (future liver remnant)

- Myrian automated software was accurate and correlated significantly with the volume of resected specimen, and hence, the estimation of FLR volume may likely correlate with the true postoperative residual liver volume. In addition, the software-based liver segmentation, FLR estimation, and color-coded three-dimensional images provide a clear road map to the surgeon to facilitate safe resection.
\end{abstract}

DOI https://doi.org/ $10.1055 / \mathrm{s}-0040-1721534$ ISSN 2457-0214.
(C)2020. Indian Society of Vascular and Interventional Radiology. This is an open access article published by Thieme under the terms of the Creative Commons Attribution-NonDerivative-NonCommercial-License, permitting copying and reproduction so long as the original work is given appropriate credit. Contents may not be used for commercial purposes, or adapted, remixed, transformed or built upon. (https://creativecommons.org/licenses/by-nc-nd/4.0/).

Thieme Medical and Scientific Publishers Pvt. Ltd. A-12, 2nd Floor, Sector 2, Noida-201301 UP, India 


\section{Introduction}

With improvements in perioperative care, liver resections are increasingly performed for primary or metastatic liver cancer, with mortality below $5 \%{ }^{1,2}$ Surgeons aim for a total resection of focal liver lesions, but they need to avoid an extensive loss of healthy liver parenchyma, since this can lead to postoperative hepatic failure. ${ }^{3}$ To perform partial liver resections safely, the determination of the entire preoperative liver volume and the remaining postoperative liver volume is important. After resection, the remnant liver must be able to preserve or recover an adequate synthetic ability to compensate for lost hepatic parenchyma. ${ }^{4}$ Thus, preoperative assessment of liver volumetry has become fundamental in selection of the patient for liver resection. With the advent of multislice computed tomography (CT), many studies have demonstrated a close correlation between intraoperative liver volume or weight measurements and virtually measured liver volumes. $^{5-8}$ Liver volume detection can be done using manual, semiautomated, and automated tracing method. Manual tracing of liver boundary on CT images though routinely used for liver volume calculation, is time consuming and prone to intra- and interobserver variations ${ }^{9}$ as compared with semiautomated liver segmentation technique. ${ }^{10}$

\section{Materials and Methods}

A single-center retrospective study of prospectively maintained data of patients who underwent anatomical liver resections between August 2012 and December 2018 was performed. Data on future liver remnant (FLR) estimation using a semiautomated software was available for 200 out of 388 patients who underwent formal hepatic resections in the given time period. The study was approved by the institutional review board. Patients who underwent nonanatomical liver resection and partial hepatectomy for hemangiomas were excluded from this study. During surgery for extracapsular excisions for hemangioma, the tumor shrinks on table during surgery and the volume of resected specimen would be lower than the estimated volume on CT scan. Hence, these patients were excluded from the study.

All patients who underwent liver resection had a triphasic CT evaluation for surgical planning. Most of the patients planned for anatomic resections were operated after estimation of resection and remnant volumes on CT. The estimated FLR was calculated using the Myrian XP-Liver (Intrasense) software by a single radiologist with more than 5 years of experience in hepatobiliary reporting, after discussing the plane of resection with the operating surgeon. The parameters evaluated by the software to estimate FLR included total liver volume, volume of normal liver to be resected, and tumor volume. The estimated resection volume, which is the sum of volume of normal liver to be resected and tumor volume, was compared with actual specimen weight measured immediately after liver resection in the operating room (OR). Surgical specimen weight was used as the gold standard and weight-to-volume ratio of 1:1 was used as a standard assumption to quantify volume of the resected specimen. The volume of resected specimen was compared with the estimated resection volume calculated using the Myrian software. The statistical analysis was performed with SPSS software version 24 (IBM Corp.).

\section{Scan Parameters and Volumetry}

Triphasic CT was performed with a multidetector CT scanner (Siemens SOMATOM Emotion 16, Siemens Healthcare $\mathrm{GmbH}$ ). Positive oral contrast-medium was not administered. After an initial noncontrast scan, triphasic CT was obtained following an intravenous administration of a iodine contrast medium at a concentration of $300 \mathrm{mg} / \mathrm{L}$ (Ultravist 300, Bayer Schering Pharma AG) through an antecubital vein of the arm, using an automatic syringe injector (Stellant, MedRad) at a flow rate of 3 to $4 \mathrm{mLs}$. The amount of contrast media used was according to the patient's weight, injecting $1.5 \mathrm{~mL} / \mathrm{kg}$. In all cases a triphasic examination was performed using a bolus tracking technique; an arterial phase scan of the upper abdomen, performed 10 seconds after the reach of the aortic enhancement threshold (100 Hounsfield unit [HU]) at the level of celiac artery; a portal phase scan of the upper abdomen, performed 35 seconds after the administration of contrast media; and a venous phase scan of the upper abdomen, performed 60 seconds after the administration of contrast media. The following parameters were used: slice thickness $1.5 \mathrm{~mm}$; increment $1.5 \mathrm{~mm}$; tube voltage $110 \mathrm{kV}$; collimation $16 \times 0.6$; pitch 1.3 ; rotation time 0.6 second.

Myrian software was used for semiautomated volumetry which allows quick automatic liver segmentation with volumetry using tissue density difference (HUs). The software allows automatic calculation of the liver vascular territories using density difference in arterial, portal, and venous phase, thus ensuring precise volumetric measurement of the liver parenchyma. Three-dimensional (3D) volumetric display

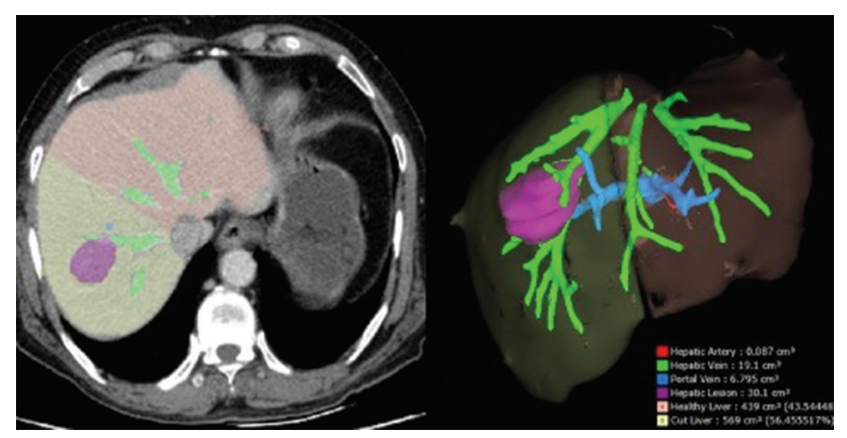

Fig. 1 A 72-year-old male with suspected malignant lesion in the right lobe of the liver was planned for right hepatectomy. (A) Cross-sectional computed tomography (CT) image shows color-coded structures-volume of liver to be resected in light green, future liver remnant in peach color (junction marks the transection plane), hepatic vein in green, and tumor in purple color. (B) Corresponding threedimensional (3D) image shows transection plane with volume of resecting segment and future liver remnant. The total volume to be resected was obtained by adding tumor volume and cut liver volume and was compared with the volume of the specimen. 
with different transparency and color levels are provided for better anatomical understanding ( - Fig. 1 ). Axial images of the arterial, portal, and venous phases were used for CT volumetry. The liver outline, hepatic artery (HA), portal vein $(\mathrm{PV})$, and hepatic veins (HV), and their branches were drawn automatically by the software. After setting seed points into the hepatocaval confluence and the main stem of the PV, the system automatically segments the HV and PV. Those vessels which were not automatically identified by the software were drawn manually. Finally, the transection plane was defined. The volumes of the intrahepatic vessels in the liver area marked for resection were included in the CT volume. After volumetric reconstruction of the normal liver parenchyma, tumor, and hepatic vasculature, virtual hepatectomy is performed in accordance with the intended surgical resection plane as decided by the operating hepatobiliary surgeon. The software automatically calculates FLR using the formula

FLR $=($ Total Liver Volume-Resected Volume $) /($ Total Liver volume-Tumor Volume)

It is expressed in percentage.

\section{Results}

Data on FLR estimation using semiautomated software was available for 200 out of 388 patients who underwent formal hepatic resections in the given time period ( - Tables $\mathbf{1}$ and $\mathbf{2}$ ). The median resected volume of surgical specimen was $650 \mathrm{~mL}$ (with interquartile range [IQR] 364-950), while median estimated volume using the Myrian software was $617 \mathrm{~mL}$ (with IQR 362-979). The mean postoperative resected volume was $801.8 \mathrm{~g}$ and the mean estimated volume was $817.57 \mathrm{~mL}$. In the evaluation of operative resection volume, Spearman's correlation test showed significant correlation between the estimated specimen volume recorded using the Myrian software with the actual value recorded in OR ( $p$-value $<0.0001)$ with correlation coefficient $(r)$ value of $0.956(\boldsymbol{- F i g}$. 2). The difference between estimated and actual specimen volume had median value of $38 \mathrm{~mL}$ (with IQR 9.25-105).

Table 1 Demographic and surgical details

\begin{tabular}{|l|l|}
\hline Age distribution & $15-79 \mathrm{y}$ \\
\hline Male:Female & $141: 59$ \\
\hline Mean age for male & $53.5 \mathrm{y}$ \\
\hline Mean age for female & $49.8 \mathrm{y}$ \\
\hline Right hepatectomy & 93 \\
\hline Right extended hepatectomy & 12 \\
\hline Right posterior sectionectomy & 7 \\
\hline Right anterior sectionectomy & 1 \\
\hline Left hepatectomy & 38 \\
\hline Left lateral hepatectomy & 31 \\
\hline Left extended hepatectomy & 8 \\
\hline Central hepatectomy & 10 \\
\hline
\end{tabular}

Table 2 Distribution of cases as per histopathology

\begin{tabular}{|l|l|}
\hline Hepatocellular carcinoma & $87(43.5 \%)$ \\
\hline Metastases & $70(35 \%)$ \\
\hline Cholangiocarcinoma & $27(13.5 \%)$ \\
\hline Hepatic adenoma & 3 \\
\hline Carcinoma gallbladder & 2 \\
\hline Angiomyolipoma & 1 \\
\hline Embryonal sarcoma & 1 \\
\hline Hepatoblastoma & 1 \\
\hline Benign biliary cystadenoma & 1 \\
\hline Pyogenic abscess & 2 \\
\hline Hydatid cyst & 2 \\
\hline Nontubercular granulomatous infection & 1 \\
\hline Tuberculosis & 1 \\
\hline Immunoglobulin G4-related pseudotumor & 1 \\
\hline
\end{tabular}

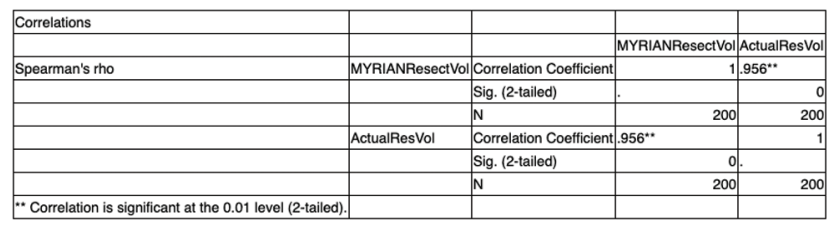

A

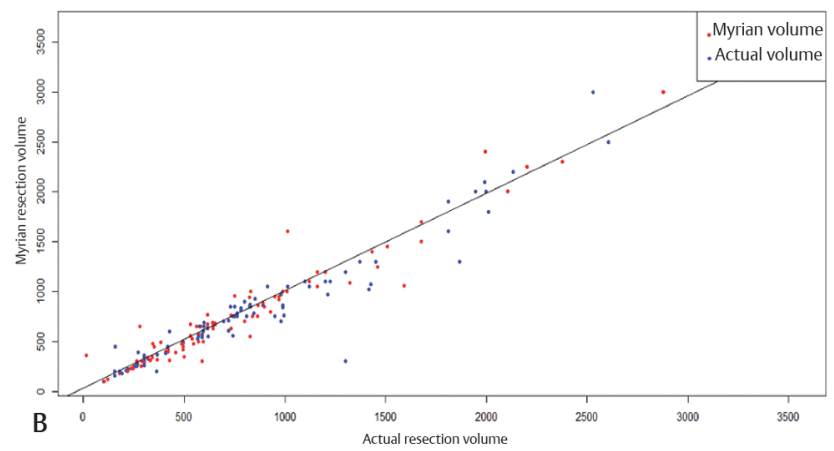

Fig. 2 Scatter plot (A) and corresponding table (B) showing correlation between estimated resection volume using semiautomated method and actual resection volume. The correlation coefficient $(r)$ is 0.956 . Red dots represent the Myrian volume and blue dots the actual volume. Volume along the $X$-axis represents specimen volume, while the $Y$-axis represents estimated volume by the Myrian software.

\section{Discussion}

Of various organs, the liver is one of the most difficult to segment virtually due to its varying shape. Ultrasound, CT, or magnetic resonance imaging (MRI) has been used to estimate hepatic volume preoperatively prior to liver resection and transplantation.

The use of CT volumetry on cadavers was first performed by Heymsfield et $\mathrm{al}^{5}$ in 1979 and the accuracy of this method was found to be within $5 \%$ of water displacement volumetry. Since then, various studies have shown preoperatively measured liver volumes using CT and MRI) correlated with intraoperative volume and weight measurement. Conversion factors have been suggested to compensate for overestimation 
of preoperative volume which could be due to blood perfusion. ${ }^{3,1}$ Median liver density estimated by intraoperative weight and volume measurement averages from 1.05 to $1.07 \mathrm{~g} / \mathrm{mL}^{11,12}$

Various automatic and semiautomatic segmentation techniques have been used to measure preoperative volumes because they had the advantage of requiring substantially lesser time compared with manual volumetry. ${ }^{13}$ Manual and automated volumetry softwares have been used for estimation of liver volumes and vascular volumes of liver donors prior to transplantation. ${ }^{9,14} \mathrm{CT}$ is more commonly used, as it is more accessible, provides higher spatial resolution, and has short acquisition time. ${ }^{9}$ Manual liver segmentation used for calculation of preoperative resection volumes however relies highly on the user performing the segmentation. ${ }^{15}$ It is done by the contouring of pixels along the boundary of the liver; or by in-painting of the liver parenchyma on sequential CT slices with the use of paintbrush tools. Once the liver has been identified on each slice, postprocessing software is used to generate liver volume. There is no exclusion of the vessels enclosed by the parenchyma in the manually painted liver. This exercise is repeated for calculation of lesion volume. The user then has to separately paint the volume of the liver which would be resected depending on the intended resection plane. This process has to be repeated many times if there are multiple possible resection planes. Manual segmentation is thus time consuming and may take up to 90 minutes per patient. ${ }^{16}$ It is also prone to intra- and interobserver variability given its inherent subjectivity. ${ }^{15}$ As a result, for a high volume center, manual segmentation is not ideally suited to guide patient selection and treatment planning.

Semiautomated and automated segmentation techniques require minimal initialization from the user; the software provides most of the optimization. The semiautomated softwares mainly employ intensity-based techniques, using density difference in arterial, portal, and venous phase allowing a precise automatic volumetric measurement of the liver parenchyma. Since the intrahepatic vessels are color coded, liver subsegmentation can be performed according to vascular supply (i.e., PVs and HAs) or drainage (i.e., HVs) rather than the standard Couinaud classification system which does not take into account the different anatomical liver variants seen in individual patients. This is especially useful for performing parenchyma sparing anatomical resections in cirrhotic patients by allowing the surgeon to remove the tumor with adequate margins without removing excess of normal liver parenchyma.

A study done for evaluation of FLR by automated and semiautomated methods in hepatectomy patients $(n=36)$ has shown the volumes using both techniques had a significant difference but a high degree of correlation with actual intraoperative specimen volume. ${ }^{17}$ Another study $(n=66)$ evaluated correlation between preoperative planned remnant liver volume and postoperative actual remnant liver volume determined from early postoperative scan using a semiautomated method and found significant correlation. ${ }^{18}$

In this study, validation of the semiautomated software was tried by comparing the preoperative estimated resection volume with the volume of the resected surgical specimen. Assuming that the density of the liver is $1 \mathrm{~kg} / \mathrm{m}^{3}$, the median resected volume of surgical specimen was found to be $650 \mathrm{~mL}$. The median estimated volume calculated using the Myrian software was $617 \mathrm{~mL}$ and the difference between estimated and actual volume had a median value of $38 \mathrm{~mL}$. Spearman's correlation test showed significant correlation ( $p$-value $<0.0001$ ) between the estimated specimen weight recorded using the Myrian software with that of the actual specimen weight with correlation coefficient $(r)$ value of 0.956 .

Liver segmentation by the Myrian software and the color-coded 3D images obtained are more comprehensible for the operating surgeon, who can easily guide or modify the resection plane. Multiple possible resection planes can be drawn and resection volumes for each can be calculated easily if there are a surgical dilemma and provide a clear road map for the surgeon to perform a safe liver resection.

Since the volume of the resected specimen closely correlates with the estimated volume estimated by Myrian software, it may be assumed that the estimated FLR would also be accurate and reliable. However, a study comparing the FLR estimated by the Myrian software with surgical outcome is required to validate this assumption.

The limitation of this study is that, the volume of surgical specimen was calculated by standard assumption of weight-to-volume ratio of liver to be 1:1 and the actual density of specimen to calculate volume was not determined. Also, the influence of cirrhosis and tumor histopathology on the density of resected specimen and volume estimation by the Myrian software was not evaluated.

\section{Conclusion}

The semiautomated software-based preoperative liver volumetry was accurate and showed significant correlation when compared with resected surgical specimen. The software-estimated FLR volume may hence correlate with the true residual liver volume and be a valuable tool to select potential surgical candidates for liver resection. The liver segmentation and color-coded 3D images provides a clear road map to the surgeon to facilitate safe resection.

\section{Conflict of Interest}

None of the authors have any conflicts of interest associated with this publication, and there has been no significant financial support for this work that could have influenced its outcome.

\section{References}

1 Poon RT, Fan ST, Lo CM, et al. Improving perioperative outcome expands the role of hepatectomy in management of benign and malignant hepatobiliary diseases: analysis of 1222 consecutive patients from a prospective database. Ann Surg 2004;240(4):698-708, discussion 708-710

2 Kamiyama T, Nakanishi K, Yokoo H, et al. Perioperative management of hepatic resection toward zero mortality and morbidity: analysis of 793 consecutive cases in a single institution. J Am Coll Surg 2010;211(4):443-449 
3 Karlo C, Reiner CS, Stolzmann P, et al. CT- and MRI-based volumetry of resected liver specimen: comparison to intraoperative volume and weight measurements and calculation of conversion factors. Eur J Radiol 2010;75(1):e107-e111

4 Michalopoulos GK, DeFrances MC. Liver regeneration. Science 1997;276(5309):60-66

5 Heymsfield SB, Fulenwider T, Nordlinger B, Barlow R, Sones P, Kutner M. Accurate measurement of liver, kidney, and spleen volume and mass by computerized axial tomography. Ann Intern Med 1979;90(2):185-187

6 Schiano TD, Bodian C, Schwartz ME, Glajchen N, Min AD. Accuracy and significance of computed tomographic scan assessment of hepatic volume in patients undergoing liver transplantation. Transplantation 2000;69(4):545-550

7 Lemke AJ, Brinkmann MJ, Schott T, et al. Living donor right liver lobes: preoperative CT volumetric measurement for calculation of intraoperative weight and volume. Radiology 2006;240(3):736-742

8 Yamanaka J, Saito S, Fujimoto J. Impact of preoperative planning using virtual segmental volumetry on liver resection for hepatocellular carcinoma. World J Surg 2007;31(6):1249-1255

9 Nakayama Y, Li Q, Katsuragawa S, et al. Automated hepatic volumetry for living related liver transplantation at multisection CT. Radiology 2006;240(3):743-748

10 Bae KT, Giger ML, Chen CT. Kahn CE Jr. Automatic segmentation of liver structure in CT images. Med Phys 1993;20(1):71-78

11 Niehues SM, Unger JK, Malinowski M, Neymeyer J, Hamm B, Stockmann M. Liver volume measurement: reason of the difference between in vivo CT-volumetry and intraoperative ex vivo determination and how to cope it. Eur J Med Res 2010;15(8):345-350

12 Lieffers JR, Mourtzakis M, Hall KD, McCargar LJ, Prado CM, Baracos VE. A viscerally driven cachexia syndrome in patients with advanced colorectal cancer: contributions of organ and tumor mass to whole-body energy demands. Am J Clin Nutr 2009;89(4):1173-1179

13 Suzuki K, Epstein ML, Kohlbrenner R, et al. Quantitative radiology: automated $\mathrm{CT}$ liver volumetry compared with interactive volumetry and manual volumetry. AJR Am J Roentgenol 2011;197(4):W706-12

14 Radtke A, Sotiropoulos GC, Nadalin S, et al. Preoperative volume prediction in adult live donor liver transplantation: 3-D CT volumetry approach to prevent miscalculations. Eur J Med Res 2008;13(7):319-326

15 Udupa JK, Leblanc VR, Zhuge Y, et al. A framework for evaluating image segmentation algorithms. Comput Med Imaging Graph 2006 30(2):75-87

16 Chartrand G, Cresson T, Chav R, Gotra A, Tang A, De Guise J. Semi-automated liver CT segmentation using Laplacian meshes. 2014 I.E. International Symposium on Biomedical Imaging, Beijing; 2013

17 Bégin A, Martel G, Lapointe R, et al. Accuracy of preoperative automatic measurement of the liver volume by CT-scan combined to a 3D virtual surgical planning software (3DVSP). Surg Endosc 2014;28(12):3408-3412

18 Simpson AL, Geller DA, Hemming AW, et al. Liver planning software accurately predicts postoperative liver volume and measures early regeneration. J Am Coll Surg 2014;219(2):199-207 